\title{
Global medical device company launches new iTero element 5D imaging system
}

Align Technology, Inc. - the global medical device company - has announced it is launching the iTero Element 5D Imaging System, which provides a new comprehensive approach to clinical applications, workflows and user experience that expands the suite of existing high-precision, full-colour imaging and fast scan times of the iTero Element portfolio.

The entire iTero product family, including the iTero Element 5D Imaging System, will be showcased at the 38th International Dental Show (IDS) 2019 in Cologne, Germany, which is running from 12 to 16 March, 2019.

In addition to offering all of the features and functionality that doctors have come to expect and rely on with the iTero Element 2 scanner, the iTero Element 5D scanner is the first integrated dental imaging system that simultaneously records $3 \mathrm{D}$, intra-oral colour and near-infrared imaging (NIRI) images and enables comparison over time using iTero's TimeLapse functionality.

Integrated 3D, intra-oral colour and NIRI technology of the iTero Element 5D Imaging System aids in detection and monitoring of interproximal caries lesions above the gingiva without using harmful radiation.

With one full arch scan, in as little as 60 seconds, the iTero Element 5D Imaging System provides doctors with powerful visualisation capabilities, including:

1. 3D impressions for restorative and orthodontic work

2. Analysis instruments, such as the occlusal clearance tool

3. NIRI imagery

4. Intraoral camera imagery

5. iTero TimeLapse technology

6. Invisalign Outcome Simulator

7. Invisalign Progress Assessment.

Zelko Relic, Align Technology, CTO and Senior Vice President for Global Research and Development said: 'The iTero Element 5D Imaging System combines the cutting-edge technology of the iTero Element portfolio of intraoral scanners, with advanced integrated imaging features, such as NIRI, which aids in interproximal caries detection and clear intra-oral images with the built-in intraoral camera.
'With this new imaging system, doctors can efficiently and effectively scan every patient at every visit and visualise treatment options together that result in more informed decisions for optimum oral care.'

Information about the iTero Element 5D Imaging System can be found at http://www.itero.com/en.
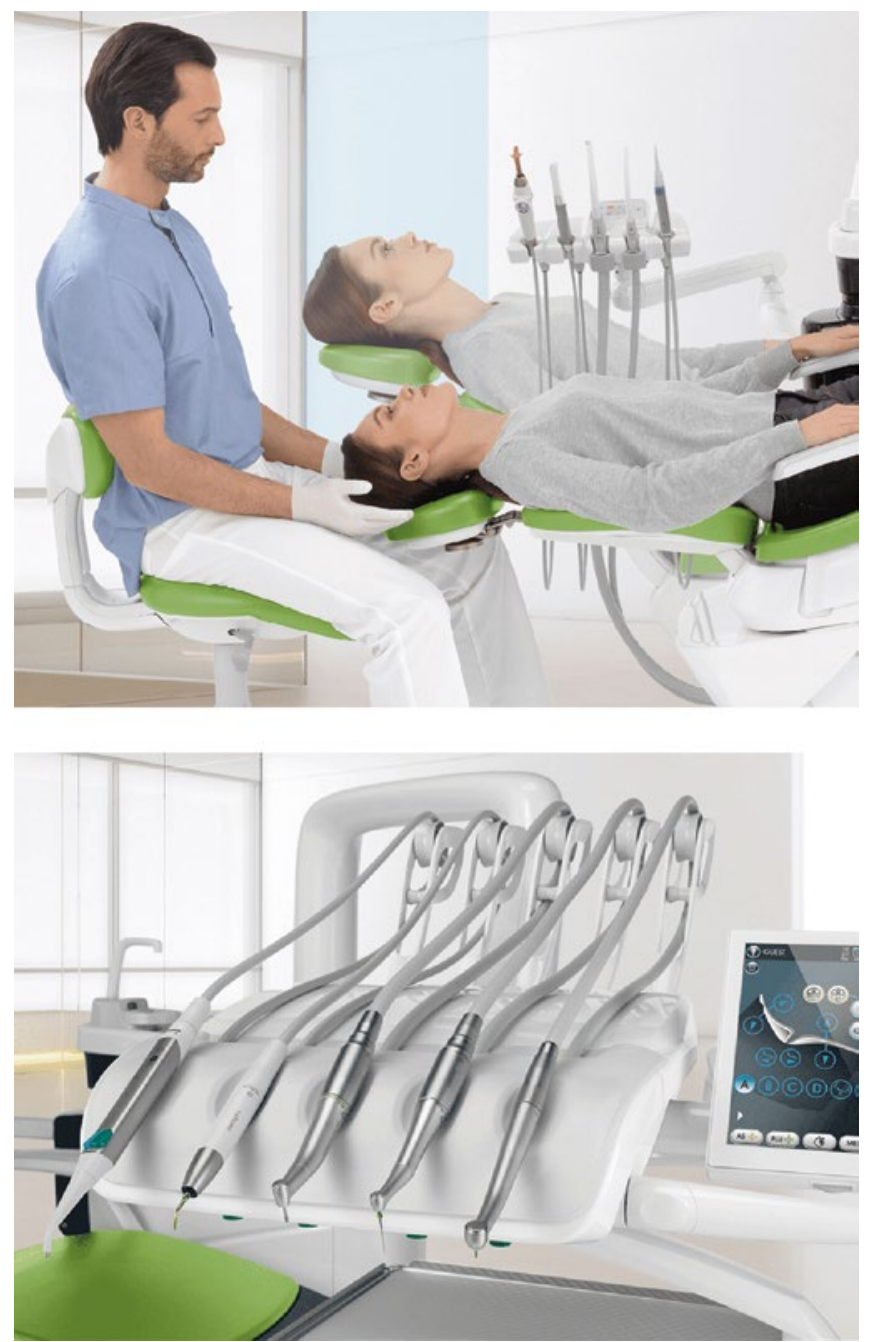

\section{Stop pathogens in their tracks}

Effective decontamination protocols and cleaning regimes are the key to preventing the spread of illnesses in dental practices, but are your cleaning products up to scratch?

Stop pathogens in their tracks by using the Steri-7 Xtra range of disinfectants from Initial Medical.

Able to deactivate $99.9999 \%$ of all pathogens, these multi-purpose cleaners can be used around the whole of a practice, providing effective defence against the spread of diseases. They also form a barrier that prevents pathogens recolonising for up to 72 hours after application.

More information is available by visiting www.initial.co.uk/

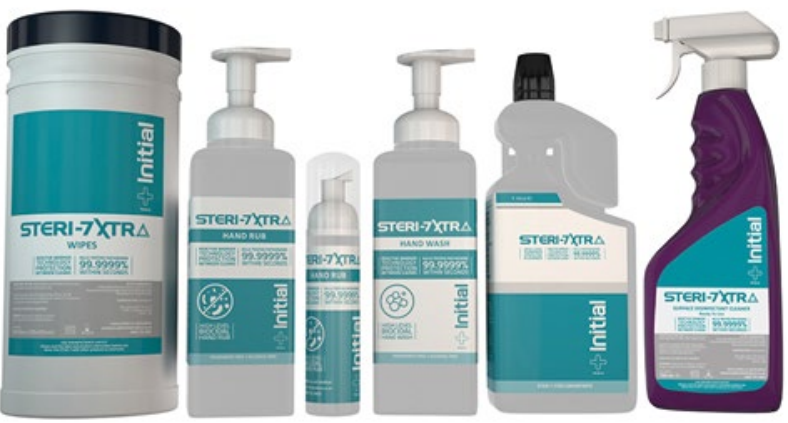
medical or calling 08708504045 . 